\title{
Biliary ascariasis - A vicious cycle
}

\author{
Nurfariza Che Husin, Ikhwan Sani Mohamad, Kah Yee Ho, Jien Yen Soh, Syed Hassan \\ Syed Aziz , Zaidi Zakaria
}

Husin N, Sani Mohamad I, Ho KY, et al. Biliary ascariasis - A vicious cycle. Malays Fam Physician. 2021;16(2);83-85. https://doi.org/10.51866/cr1078

Keywords:

Biliary, ascariasis, cholangitis, ERCP

\section{Authors:}

\author{
Ikhwan Sani Mohamad \\ (Corresponding author) \\ Department of Surgery, School of \\ Medical Sciences, Universiti Sains \\ Malaysia, Kelantan, Malaysia \\ Email: ikhwansani@yahoo.com.my
}

Hospital Universiti Sains Malaysia Kelantan, Malaysia

\section{Nurfariza Che Husin}

Department of Surgery, School of

Medical Sciences, Universiti Sains

Malaysia, Kelantan, Malaysia

Hospital Universiti Sains Malaysia

Kelantan, Malaysia

\section{Ho Kah Yee}

Department of Surgery, School of Medical Sciences, Universiti Sains Malaysia, Kelantan, Malaysia

Hospital Universiti Sains Malaysia Kelantan, Malaysia

\section{Soh Jien Yen}

Department of Surgery, School of Medical Sciences, Universiti Sains Malaysia, Kelantan, Malaysia

Hospital Universiti Sains Malaysia Kelantan, Malaysia

\section{Syed Hassan Syed Aziz}

Department of Surgery, School of Medical Sciences, Universiti Sains Malaysia, Kelantan, Malaysia

Hospital Universiti Sains Malaysia Kelantan, Malaysia

\begin{abstract}
Biliary ascariasis is a rare disease in a non-endemic area. However, it is one of the possible etiological factors for retarded growth as well as malnutrition in children. It may cause intestinal obstruction, appendicitis, biliary obstruction, liver abscess, hepatolithiasis, and pancreatitis in adults. Herein, we report a patient with ascending cholangitis secondary to biliary ascariasis who was successfully managed with Endoscopic Retrograde Cholangio Pancreaticography.
\end{abstract}

\section{Introduction}

The most common causative agent of ascariasis is Ascaris lumbricoides. Ascariasis may exist as a zoonotic infection associated with pigs, but in most endemic areas it is transmitted via fecal-oral or direct person-to-person contact. ${ }^{1,2}$ This infection is usually asymptomatic. Biliary ascariasis is a rare disease, especially in non-endemic areas. Hepatobiliary ascariasis is a result of migration of the worm into the biliary tree, resulting in obstructive symptoms. ${ }^{3}$ Conservative management may be applied, but if symptoms worsen or persist, endoscopic extraction of the worms is the main treatment. Inadequate extraction or remnants of the parasites left within the biliary tree may produce unfavorable outcomes and may result in oriental cholangiohepatitis if left untreated. ${ }^{3}$ Although ascariasis is a common helminthic infestation in Malaysia, biliary ascariasis is rarely reported.

\section{Case presentation}

A 68-year-old woman presented with epigastric pain and vomiting for one week. The pain was described as dull and aching in nature, associated with fever, yellowish discoloration of skin, tea colored urine, and pale stool. She also had a history of passing worms in her stool. There was a history of multiple episodes of gallstone pancreatitis and symptomatic hepatolithiasis in the right lobe of the liver over a 10 -year period that was investigated by serial ultrasounds and CT scans. She underwent cholecystectomy for gallstones during the fifth year of treatment but refused other surgery-based treatment for hepatolithiasis. The patient was averagely built and well-nourished but appeared jaundiced. There was tenderness in the epigastric region as well as the right hypochondrium. Blood investigations revealed leukocytosis with conjugated hyperbilirubinemia. The latest ultrasonography showed persistent hyperechoic linear structure suggestive of worms in the common bile duct causing biliary obstruction (Figure 1). Endoscopic Retrograde Cholangio Pancreaticography (ERCP) was performed and showed multiple filling defects at the common bile duct and intrahepatic ducts. There were multiple small stones with clumps of worms after endoscopic sphincterotomy and balloon trawling were performed (Figures 2 and 3). Subsequently, she was treated with intravenous fluids and oral albendazole (antihelminth) $400 \mathrm{mg}$ daily for three days. The patient was discharged home after one week of hospital stay and was given regular out-patient follow up with serial ultrasonography.

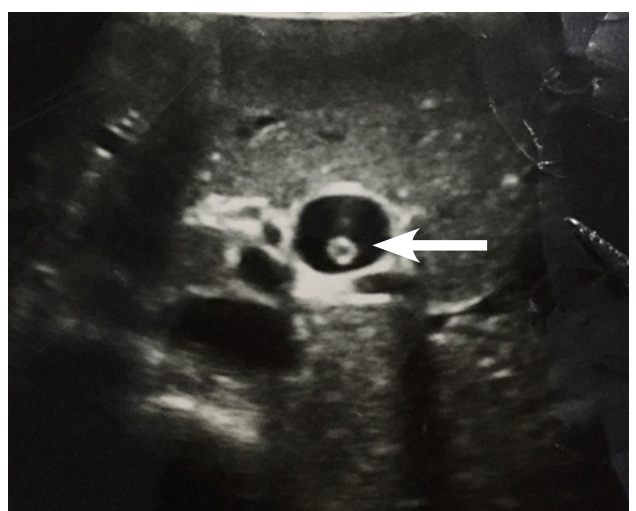

Figure 1: Ultrasonography finding showed dilated common bile duct with presence of ascariasis (arrow) 


\section{Zaidi Zakaria}

Department of Surgery, School of Medical Sciences, Universiti Sains Malaysia, Kelantan, Malaysia

Hospital Universiti Sains Malaysia Kelantan, Malaysia

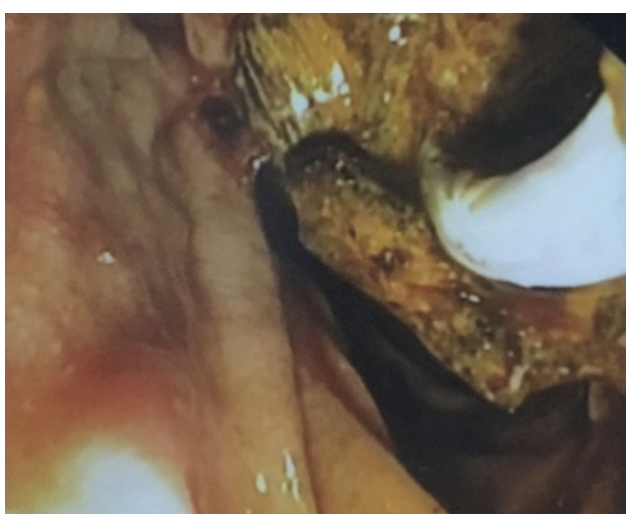

Figure 2: Endoscopic image during ERCP showed extraction of soft stones using balloon extractor

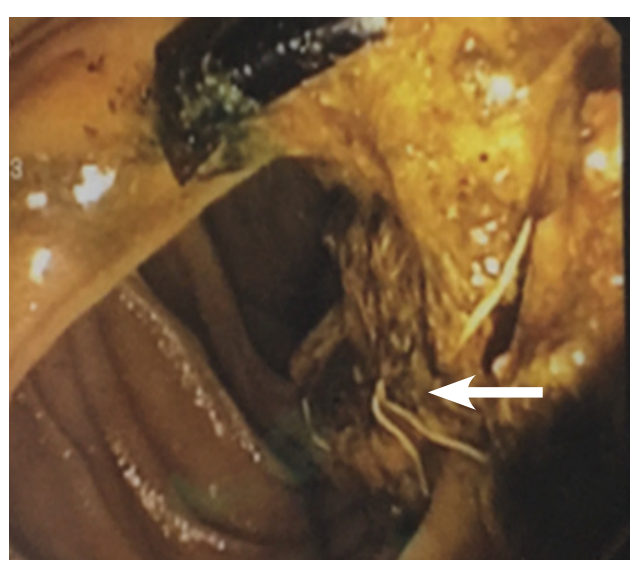

Figure 3: Endoscopic image during ERCP showed multiple worms were extracted from the common bile duct (arrow)

\section{Discussion}

Ascariasis is a common helminthic infection in Malaysia, mostly in rural areas. The most common species is Ascaris lumbricoides. People get infected primarily by fecal-oral transmission. The worm egg will become a larva that normally lives in the small intestines, mainly in the terminal ileum. The lifespan of adult worms is only 1-2 years in the human intestines. Persistent infection occurs as a result of frequent re-exposure and reinfection of the host. ${ }^{1}$

The worms are able to pass through the intestinal wall and migrate through the portal system to the liver and lung. ${ }^{1}$ Complications may arise when the worms migrate into the bile duct and liver. This condition will lead to biliary colic, cholangitis, biliary obstruction, pancreatitis, and liver abscess as well as hepatolithiasis in chronic worm infestation. ${ }^{1,3}$

Patients will usually present with malnutrition, chronic anemia, fever, jaundice, leukocytosis, and abdominal tenderness (especially at the right upper quadrant, lower quadrant, and epigastric region). The diagnosis of biliary ascariasis is based on history taking, physical examination, stool ova, and cysts, as well as ultrasonography or CT scan of the hepatobiliary system. The sonographic appearance in biliary ascariasis are long linear or curved echogenic structures without acoustic shadow or a central, longitudinal anechoic tube, likely representing the digestive tract of the worm. ${ }^{1,3,4}$

ERCP is the best modality to demonstrate worms in the biliary tract. However, this procedure also has its limitations, as it must be done during active biliary symptoms as worms will migrate out of the bile duct. ERCP is only performed once the diagnosis is established by other minimally invasive modalities such as ultrasonography, CT scan, or even Magnetic Resonance Cholangiopancreatography (MRCP) to avoid unnecessary complications. ${ }^{4}$

The treatment of biliary ascariasis should be a holistic and multidisciplinary approach. It starts with prevention, such as a community deworming program, public awareness, and health and hygiene education by public health officers to increase awareness in the public, followed by adequate nutritional support to enhance immunity toward worm infection. The best medical therapies need to be given adequately to achieve at least $95.7 \%$ cure rates. ${ }^{1}$ Oral medications are albendazole $400 \mathrm{mg}$ single dose for uncomplicated infection or mebendazole $100 \mathrm{mg}$ BID for three days for ascariasis with coexisting whipworm infection. Another alternative drug is ivermectin 150$200 \mathrm{mcg} / \mathrm{kg}$ single dose.

Patients from endemic areas or with risk of reexposure and reinfection may still be carrying larvae. They should be re-evaluated in three months and retreated if stool ova persist. ${ }^{1}$ ERCP plays an important role for therapeutic treatment in biliary ascariasis. The worms can be removed from the ampulla of Vater using a Dormia basket. ERCP is a good alternative to open surgery as it reduces morbidity, hospital stay, and wound complications. ${ }^{5}$

\section{Conclusion}

Biliary ascariasis is a treatable disease. Although it is uncommon, it should be considered in patients with recurrent biliary colic, liver abscess, and cholangitis from endemic areas or tropical countries. Treatment includes oral antihelminthic and endoscopic or surgical removal of worms. 


\section{References}

1. David R Haburchak. Ascariasis. Emedicine. 2018 Jul 30. Available from: https:// emedicine.medscape.com/article/212510overview

2. Zachary A. Silver, Saravanakumar P. Kaliappan. Geographical distribution of soil transmitted helminths and the effects of community type in South Asia and South East Asia - A systematic review. PLoS Negl Trop Dis. 2018 Jan; 12(1): e0006153. Available from: https://journals.plos.org/plosntds/ article?id=10.1371/journal.pntd.0006153
3. S Misra, M. Dwivedi. Clinical features and management of biliary ascariasis in a non-endemic area. Postgrad Med J. 2000 Jan; 76(891): 29-32. doi: 10.1136/pmj.76.891.29. Available from: https://www.ncbi.nlm.nih.gov pmc/articles/PMC1741472/

4. Mohammad Sultan Khuroo, Showkat Ali Zargar, Rakesh Mahajan, Roshan Lal Bhat, Gul Javid. Sonographic appearances in biliary ascariasis. Gastroenterology. August 1987;93(2): Pages 267-272. Available from: https://www.gastrojournal.org/ article/0016-5085(87)91013-4/fulltext
5. Towhidul Alam. ERCP in the Management of Biliary Ascariasis. SAGES. Program Number: P220. Available from: https://www.sages. org/meetings/annual 132 meeting/abstractsarchive/ercp-in-the118 management-ofbiliary-ascariasis 